\title{
Research Article \\ Strong Convergence Theorems for Countable Lipschitzian Mappings and Its Applications in Equilibrium and Optimization Problems
}

\author{
Liping Yang ${ }^{1}$ and Yongfu $\mathrm{Su}^{2}$ \\ ${ }^{1}$ Department of Fundamental, Tianjin Institute of Urban Construction, Tianjin 300384, China \\ ${ }^{2}$ Department of Mathematics, Tianjin Polytechnic University, Tianjin 300160, China
}

Correspondence should be addressed to Yongfu Su, suyongfu@tjpu.edu.cn

Received 21 October 2008; Revised 20 December 2008; Accepted 5 March 2009

Recommended by Naseer Shahzad

The purpose of this paper is to propose a modified hybrid method in mathematical programming and to obtain some strong convergence theorems for common fixed points of a countable family of Lipschitzian mappings. Further, we apply our results to solve the equilibrium and optimization problems. The results of this paper improved and extended the results of W. Nilsrakoo and S. Saejung (2008) and some others in some respects.

Copyright (C) 2009 L. Yang and Y. Su. This is an open access article distributed under the Creative Commons Attribution License, which permits unrestricted use, distribution, and reproduction in any medium, provided the original work is properly cited.

\section{Introduction and Preliminaries}

Let $H$ be a real Hilbert space with inner product $\langle\cdot, \cdot\rangle$ and norm $\|\cdot\|$ and let $C$ be a nonempty subset of $H$. A mapping $T: C \rightarrow C$ is said to be Lipschitzian if there exists a positive constant $L$ such that

$$
\|T x-T y\| \leq L\|x-y\|, \quad \forall x, y \in C .
$$

In this case, $T$ is also said to be $L$-Lipschitzian. Throughout the paper, we assume that every Lipschitzian mapping is $L$-Lipschitzian with $L \geq 1$. If $L=1$, then $T$ is known as a nonexpansive mapping. We denote by $F(T)$ the set of fixed points of $T$. There are many methods for approximating the fixed points of a nonexpansive mapping. In 1953, Mann [1] introduced the following iteration method:

$$
x_{n+1}=\alpha_{n} x_{n}+\left(1-\alpha_{n}\right) T x_{n}
$$


where the initial guess element $x_{0} \in C$ is arbitrary, and $\left\{\alpha_{n}\right\}$ is a real sequence in $[0,1]$. Mann iteration has been extensively investigated for nonexpansive mappings. One of the fundamental convergence results is proved by Qin and Su [2]. In an infinite-dimensional Hilbert space, Mann iteration could conclude only weak convergence [3]. Attempts to modify the Mann iteration method (1.2) so that strong convergence is guaranteed have recently been made. Nakajo and Takahashi [4] proposed the following modification of Mann iteration $\operatorname{method}(1.2)$ :

$$
\begin{gathered}
x_{0} \in C \text { chosen arbitrarily, } \\
y_{n}=\alpha_{n} x_{n}+\left(1-\alpha_{n}\right) T x_{n}, \\
C_{n}=\left\{z \in C:\left\|y_{n}-z\right\| \leq\left\|x_{n}-z\right\|\right\}, \\
Q_{n}=\left\{z \in C:\left\langle x_{n}-z, x_{0}-x_{n}\right\rangle \geq 0\right\}, \\
x_{n+1}=P_{C_{n} \cap Q_{n}}\left(x_{0}\right),
\end{gathered}
$$

where $P_{K}$ denotes the metric projection from $H$ onto a closed convex subset $K$ of $H$. The iterative method (1.3) is said to be hybrid method or $C Q$ method. In recent years, the hybrid method (1.3) has been modified by many authors for other nonlinear operators [2, 5-10].

In 2008, Nilsrakoo and Saejung [11] used the hybrid method to obtain a strong convergence theorem for countable Lipschitzian mappings $\left\{T_{n}\right\}_{n=0}^{\infty}$ as follows.

Theorem NS. Let $C$ be a nonempty bounded closed convex subset of a real Hilbert space $H$. Let $\left\{T_{n}\right\}_{n=0}^{\infty}$ be a sequence of $L_{n}$-Lipschitzian mappings from $C$ into itself with $L_{n} \geq 1$ and let $\bigcap_{n=0}^{\infty} F\left(T_{n}\right)$ be nonempty. Assume that $\left\{\alpha_{n}\right\}$ is a sequence in $[0,1)$ with $\lim _{\sup _{n \rightarrow \infty}} \alpha_{n}<1$. Let $\left\{x_{n}\right\}$ be a sequence in $C$ defined as follows:

$$
\begin{gathered}
x_{0} \in C \text { chosen arbitrarily, } \\
y_{n}=\alpha_{n} x_{n}+\left(1-\alpha_{n}\right) T_{n} x_{n}, \\
C_{n}=\left\{z \in C:\left\|y_{n}-z\right\|^{2} \leq\left\|x_{n}-z\right\|^{2}+\theta_{n}\right\}, \\
Q_{n}=\left\{z \in C:\left\langle x_{n}-z, x_{0}-x_{n}\right\rangle \geq 0\right\}, \\
x_{n+1}=P_{C_{n} \cap Q_{n}}\left(x_{0}\right),
\end{gathered}
$$

where

$$
\theta_{n}=\left(1-\alpha_{n}\right)\left(L_{n}^{2}-1\right)(\operatorname{diam} C)^{2} \longrightarrow 0,
$$

as $n \rightarrow \infty$. Let $\sum_{n=1}^{\infty} \sup \left\{\left\|T_{n+1} z-T_{n} z\right\|: z \in B\right\}<\infty$ for any bounded subset $B$ of $C$, and let $T$ be a mapping of $C$ into itself defined by $T z=\lim _{n \rightarrow \infty} T_{n} z$, for all $z \in C$ and suppose that $F(T)=\bigcap_{n=0}^{\infty} F\left(T_{n}\right)$, then $\left\{x_{n}\right\}$ converges strongly to $P_{F(T)} x_{0}$.

Nilsrakoo and Saejung also apply the aforementioned result to obtain an applied result for equilibrium problems. 
The purpose of this paper is to propose a modified hybrid method in mathematical programming and to obtain some strong convergence theorems for common fixed points of a countable family of Lipschitzian mappings. Finally, we apply our results to solve the equilibrium and optimization problems. The results of this paper improved and extended the Nilsrakoo and Saejung results in some respects.

Recall that given a closed convex subset $K$ of a real Hilbert space $H$, the nearest point projection $P_{K}$ from $H$ onto $K$ assigns to each $x \in H$ its nearest point denoted by $P_{K} x$ in $K$ from $x$ to $K$; that is, $P_{K} x$ is the unique point in $K$ with the property

$$
\left\|x-P_{K} x\right\| \leq\|x-y\| \quad \forall y \in K .
$$

The following lemma is well known.

Lemma 1.1. Let $K$ be a closed convex subset of a real Hilbert space $H$. Given $x \in H$ and $z \in K$. Then $z=P_{K} x$ if and only if there holds the relation:

$$
\langle x-z, y-z\rangle \leq 0 \quad \forall y \in K
$$

Definition 1.2. Let $C$ be a nonempty closed convex subset of a real Hilbert space $H$ and let $\left\{T_{n}\right\}_{n=0}^{\infty}$ be a sequence of $L_{n}$-Lipschitzian mappings from $C$ into itself with $L_{n} \geq 1 .\left\{T_{n}\right\}_{n=0}^{\infty}$ is said to satisfy the (SU) condition, if the following conditions hold:

(1) for any strong convergence sequence $\left\{x_{n}\right\} \subset C$, the sequence $\left\{T_{n} x_{n}\right\}$ is also strong convergent;

(2) the common fixed points set $\bigcap_{n=0}^{\infty} F\left(T_{n}\right)$ is nonempty;

(3) $F(T)=\bigcap_{n=0}^{\infty} F\left(T_{n}\right)$, where $T: C \rightarrow C$ is defined by $T x=\lim _{n \rightarrow \infty} T_{n} x$, for all $x \in C$, $F(T)$ denotes the fixed points set of $T$.

In Section 3 of this paper, we will give an important example of sequence of $L_{n^{-}}$ Lipschitzian mappings which satisfies the (SU) condition.

Lemma 1.3. Let $C$ be a nonempty closed convex subset of a real Hilbert space $H$ and let $\left\{T_{n}\right\}_{n=0}^{\infty}$ be a sequence of $L_{n}$-Lipschitzian mappings from $C$ into itself with $L_{n} \geq 1$. If $\left\{T_{n}\right\}_{n=0}^{\infty}$ satisfies the (SU) condition. Then

(1) $\left\{L_{n}\right\}$ is bounded implies $T$ is uniformly continuous on the $C$;

(2) $\lim _{n \rightarrow \infty} L_{n}=L$ implies $T$ is $L$-Lipschitzian;

(3) $\lim _{n \rightarrow \infty} L_{n}=1 \mathrm{implies} T$ is nonexpansive.

Proof. Observe that, for all $x, y \in C$, we have

$$
\|T x-T y\|=\lim _{n \rightarrow \infty}\left\|T_{n} x-T_{n} y\right\| \leq \lim _{n \rightarrow \infty} L_{n}\|x-y\|
$$

The results (1)-(3) are easy to prove. 


\section{Main Results}

Theorem 2.1. Let $C$ be a nonempty closed convex subset of a real Hilbert space $H$. Let $\left\{T_{n}\right\}_{n=0}^{\infty}$ be a sequence of $L_{n}$-Lipschitzian mappings from $C$ into itself with $L_{n} \rightarrow 1$, as $n \rightarrow \infty$. Assume $\left\{T_{n}\right\}_{n=0}^{\infty}$ satisfies the (SU) condition and $\left\{\alpha_{n}\right\}$ is a sequence in $[0,1)$ with $\limsup _{n \rightarrow \infty} \alpha_{n}<1$. Let $\left\{x_{n}\right\}_{n=0}^{\infty}$ be a sequence in $C$ defined as follows:

$$
\begin{gathered}
x_{0} \in C \text { chosen arbitrarily, } \\
y_{n}=\alpha_{n} x_{n}+\left(1-\alpha_{n}\right) T_{n} x_{n}, \\
C_{n}=\left\{z \in C_{n-1} \bigcap Q_{n-1}:\left\|y_{n}-z\right\| \leq\left\|x_{n}-z\right\|+\theta_{n}\right\}, \quad n \geq 1, \\
C_{0}=\left\{z \in C:\left\|y_{0}-z\right\| \leq\left\|x_{0}-z\right\|+\theta_{0}\right\}, \\
Q_{n}=\left\{z \in C_{n-1} \bigcap Q_{n-1}:\left\langle x_{n}-z, x_{0}-x_{n}\right\rangle \geq 0\right\}, \quad n \geq 1, \\
Q_{0}=C \\
x_{n+1}=P_{C_{n} \cap Q_{n}}\left(x_{0}\right),
\end{gathered}
$$

where

$$
\begin{array}{r}
\theta_{n}=\left(1-\alpha_{n}\right)\left(L_{n}^{2}-1\right)\left(\sup _{z \in A}\left\|x_{n}-z\right\|\right)^{2}, \\
A=\left\{y \in F(T):\left\|y-P_{F(T)} x_{0}\right\| \leq 1\right\} .
\end{array}
$$

Then $\left\{x_{n}\right\}$ converges strongly to $P_{F(T)} x_{0}$, where $P_{K}$ is the metric projection from $H$ onto closed convex subset $K$.

Proof. We first prove that $C_{n}$ and $Q_{n}$ are closed and convex for each $n \geq 0$. From the definition of $C_{n}$ and $Q_{n}$, it is obvious that $C_{n}$ is closed and $Q_{n}$ is closed and convex for each $n \geq 0$. We prove that $C_{n}$ is convex. Since $\left\|y_{n}-z\right\| \leq\left\|x_{n}-z\right\|+\theta_{n}$ is equivalent to

$$
2\left\langle x_{n}-y_{n}, z\right\rangle \leq\left\|x_{n}\right\|^{2}-\left\|y_{n}\right\|^{2}+\theta_{n}
$$

it follows that $C_{n}$ is convex. Next, we show that $F(T) \subset C_{n}$, for all $n \geq 0$. For any $p \in F(T)$, we have

$$
\begin{aligned}
\left\|y_{n}-p\right\|^{2} & =\left\|\alpha_{n} x_{n}+\left(1-\alpha_{n}\right) T_{n} x_{n}-p\right\|^{2} \\
& \leq \alpha_{n}\left\|x_{n}-p\right\|^{2}+\left(1-\alpha_{n}\right)\left\|T_{n} x_{n}-p\right\|^{2} \\
& =\alpha_{n}\left\|x_{n}-p\right\|^{2}+\left(1-\alpha_{n}\right) L_{n}^{2}\left\|x_{n}-p\right\|^{2} \\
& \leq\left\|x_{n}-p\right\|^{2}+\left(1-\alpha_{n}\right)\left(L_{n}^{2}-1\right)\left\|x_{n}-p\right\|^{2} \\
& \leq\left\|x_{n}-p\right\|^{2}+\theta_{n},
\end{aligned}
$$


so that $p \in C_{n}$, therefore, we have $F(T) \subset C_{n}$, for all $n \geq 0$. Next, we show that $F(T) \subset Q_{n}$, for all $n \geq 0$. We prove this by induction. For $n=0$, we have $F(T) \subset C=Q_{0}$. Suppose that $F(T) \subset Q_{n}$, then $F(T) \subset C_{n} \cap Q_{n}$ and there exists a unique element $x_{n+1} \in C_{n} \cap Q_{n}$ such that $x_{n+1}=P_{C_{n} \cap Q_{n}} x_{0}$. By using Lemma 1.1, we have

$$
\left\langle x_{n+1}-z, x_{0}-x_{n+1}\right\rangle \geq 0,
$$

for all $z \in C_{n} \cap Q_{n}$. In particular,

$$
\left\langle x_{n+1}-p, x_{0}-x_{n+1}\right\rangle \geq 0
$$

for all $p \in F(T)$. It follows from the definition of $Q_{n+1}$ that $F(T) \subset Q_{n+1}$. By using the principle of induction, we claim that $F(T) \subset Q_{n}$, for all $n \geq 0$. Therefore, we have $F(T) \subset C_{n} \cap Q_{n}$, for all $n \geq 0$. Now the sequence $\left\{x_{n}\right\}$ is well defined.

It follows from the definition of $Q_{n}$ that $x_{n}=P_{Q_{n}} x_{0}$. Therefore, we have

$$
\left\|x_{n}-x_{0}\right\| \leq\left\|z-x_{0}\right\|
$$

for all $z \in F(T) \subset Q_{n}$. This implies that the $\left\{\left\|x_{n}-x_{0}\right\|\right\}$ is bounded. On the other hand, from $x_{n+1}=P_{C_{n} \cap Q_{n}} x_{0} \in Q_{n}$, we have

$$
\left\|x_{n}-x_{0}\right\| \leq\left\|x_{n+1}-x_{0}\right\|,
$$

for all $n \geq 0$. Therefore, $\left\{\left\|x_{n}-x_{0}\right\|\right\}$ is nondecreasing and bounded. So that $\lim _{n \rightarrow \infty}\left\|x_{n}-x_{0}\right\|$ exists.

Note again that $x_{n}=P_{Q_{n}} x_{0}$, hence for any positive integer $m$, we have $x_{n+m} \in Q_{n+m-1} \subset$ $Q_{n}$ which implies that $\left\langle x_{n+m}-x_{n}, x_{n}-x_{0}\right\rangle \geq 0$. Therefore, we have

$$
\begin{aligned}
\left\|x_{n+m}-x_{n}\right\|^{2} & =\left\|\left(x_{n+m}-x_{0}\right)-\left(x_{n}-x_{0}\right)\right\|^{2} \\
& =\left\|x_{n+m}-x_{0}\right\|^{2}-\left\|x_{n}-x_{0}\right\|^{2}-2\left\langle x_{n+m}-x_{n}, x_{n}-x_{0}\right\rangle \\
& \leq\left\|x_{n+m}-x_{0}\right\|^{2}-\left\|x_{n}-x_{0}\right\|^{2} .
\end{aligned}
$$

From this inequality, we know that $\left\{x_{n}\right\}$ is a Cauchy sequence in $C$, so that there exists a point $p \in C$ such that $\lim _{n \rightarrow \infty} x_{n}=p$.

Since $x_{n+1} \in C_{n}$, then $\left\|y_{n}-x_{n+1}\right\|^{2} \leq\left\|x_{n}-x_{n+1}\right\|^{2}+\theta_{n}$ this together with $\theta_{n} \rightarrow 0$, as $\rightarrow \infty$, implies that $\lim _{n \rightarrow \infty} y_{n}=p$. From $\lim \sup _{n \rightarrow \infty} \alpha_{n}<1$, we get

$$
\left\|x_{n}-T_{n} x_{n}\right\| \leq \frac{1}{1-\alpha_{n}}\left\|y_{n}-x_{n}\right\| \longrightarrow 0
$$


as $n \rightarrow \infty$. Since $T$ is nonexpansive, therefore,

$$
\begin{aligned}
\left\|x_{n}-T x_{n}\right\| & \leq\left\|x_{n}-T_{n} x_{n}\right\|+\left\|T_{n} x_{n}-T x_{n}\right\| \\
& \leq\left\|x_{n}-T_{n} x_{n}\right\|+\left\|T_{n} x_{n}-T_{n} p\right\|+\left\|T_{n} p-T p\right\|+\left\|T p-T x_{n}\right\| \\
& \leq\left\|x_{n}-T_{n} x_{n}\right\|+L_{n}\left\|x_{n}-p\right\|+\left\|T_{n} p-T p\right\|+\left\|p-x_{n}\right\| \longrightarrow 0,
\end{aligned}
$$

as $n \rightarrow \infty$. Then $\lim _{n \rightarrow \infty} x_{n}=p \in F(T)$.

Finally, we claim that $p=p_{0}=P_{F(T)} x_{0}$. If not, we have $\left\|x_{0}-p\right\|>\left\|x_{0}-p_{0}\right\|$. There must exists a positive integer $N$, if $n>N$, then $\left\|x_{0}-x_{n}\right\|>\left\|x_{0}-p_{0}\right\|$, which leads to

$$
\begin{aligned}
\left\|x_{0}-p_{0}\right\|^{2} & =\left\|x_{0}-x_{n}+x_{n}-p_{0}\right\|^{2} \\
& =\left\|x_{0}-x_{n}\right\|^{2}+\left\|x_{n}-p_{0}\right\|^{2}+2\left\langle x_{n}-p_{0}, x_{0}-x_{n}\right\rangle .
\end{aligned}
$$

It follows that $\left\langle x_{n}-p_{0}, x_{0}-x_{n}\right\rangle<0$ implies that $p_{0} \notin Q_{n}$ This is a contradiction, hence $p=p_{0}$. This completes the proof.

The following theorem directly follows from Theorem 2.1.

Theorem 2.2. Let $C$ be a nonempty closed convex subset of a real Hilbert space $H$. Let $\left\{T_{n}\right\}_{n=0}^{\infty}$ be a sequence of nonexpansive mappings from $C$ into itself. Assume $\left\{T_{n}\right\}_{n=0}^{\infty}$ satisfies the (SU) condition and $\left\{\alpha_{n}\right\}$ is a sequence in $[0,1)$ with $\limsup _{n \rightarrow \infty} \alpha_{n}<1$. Let $\left\{x_{n}\right\}_{n=0}^{\infty}$ be a sequence in $C$ defined as follows:

$$
\begin{gathered}
x_{0} \in C \text { chosen arbitrarily, } \\
y_{n}=\alpha_{n} x_{n}+\left(1-\alpha_{n}\right) T_{n} x_{n}, \\
C_{n}=\left\{z \in C_{n-1} \bigcap Q_{n-1}:\left\|y_{n}-z\right\| \leq\left\|x_{n}-z\right\|\right\}, \quad n \geq 1, \\
C_{0}=\left\{z \in C:\left\|y_{0}-z\right\| \leq\left\|x_{0}-z\right\|\right\}, \\
Q_{n}=\left\{z \in C_{n-1} \bigcap Q_{n-1}:\left\langle x_{n}-z, x_{0}-x_{n}\right\rangle \geq 0\right\}, \quad n \geq 1, \\
Q_{0}=C, \\
x_{n+1}=P_{C_{n} \cap Q_{n}}\left(x_{0}\right) .
\end{gathered}
$$

Then $\left\{x_{n}\right\}$ converges strongly to $P_{F(T)} x_{0}$. Where $P_{K}$ is the metric projection from $H$ onto closed convex subset $K$. 


\section{Application for Equilibrium and Optimization}

Let $C$ be a nonempty closed convex subset of a real Hilbert space $H$. Let $f$ be a bifunction of $C \times C$ into $\mathbf{R}$, where $\mathbf{R}$ is the set of real numbers. The equilibrium problem for $F: C \times C \rightarrow \mathbf{R}$ is to find $x \in C$ such that

$$
f(x, y) \geq 0, \quad \forall y \in C
$$

The set of solutions of (3.1) is denoted by $\mathrm{EP}(F)$. Given a mapping $T: C \rightarrow H$, let $f(x, y)=$ $\langle T x, y-x\rangle$, for all $x, y \in C$. Then, $z \in \operatorname{EP}(f)$ if and only if $\langle T z, y-z\rangle \geq 0$, for all $y \in C$, that is, $z$ is a solution of the variational inequality. Numerous problems in physics, optimization, and economics reduce to find a solution of (3.1). Some methods have been proposed to solve the equilibrium problem; see, for instance, [12-16].

For solving the equilibrium problem for a bifunction $f: C \times C \rightarrow \mathbf{R}$, let us assume that $f$ satisfies the following conditions:

(A1) $f(x, x)=0$, for all $x \in C$;

(A2) $f$ is monotone, that is, $f(x, y)+f(y, x) \leq 0$, for all $x, y \in C$;

(A3) for each $x, y, z \in C$,

$$
\lim _{t \downarrow 0} f(t z+(1-t) x, y) \leq f(x, y)
$$

(A4) for each $x \in C, y \mapsto f(x, y)$ is convex and lower semicontinuous.

We need the following lemmas for the proof of our main results.

Lemma 3.1 (see [11-13]). Let $C$ be a nonempty closed convex subset of $H$ and let $f$ be a bifunction of $C \times C$ into $\mathbf{R}$ satisfying (A1)-(A4). Let $r>0$ and $x \in H$. Then, there exists $z \in C$ such that

$$
f(z, y)+\frac{1}{r}\langle y-z, z-x\rangle \geq 0, \quad \forall y \in C
$$

Lemma 3.2 (see [11-13]). Assume that $f: C \times C \rightarrow \mathbf{R}$ satisfies (A1)-(A4). For $r>0$ and $x \in H$, define a mapping $T_{r}: H \rightarrow C$ as follows:

$$
T_{r}(x)=\left\{z \in C: f(z, y)+\frac{1}{r}\langle y-z, z-x\rangle \geq 0, \forall y \in C\right\}
$$

for all $z \in H$. Then, the following hold:

(1) $T_{r}$ is single-valued;

(2) $T_{r}$ is firmly nonexpansive, that is, for any $x, y \in H$,

$$
\left\|T_{r} x-T_{r} y\right\|^{2} \leq\left\langle T_{r} x-T_{r} y, x-y\right\rangle
$$


(3) $F\left(T_{r}\right)=\mathrm{EP}(f)$;

(4) $\mathrm{EP}(f)$ is closed and convex.

Remark 3.3. $T_{r}$ is also nonexpansive, for all $r>0$.

Now, we prove the following lemma which is very important for the main results of this section.

Lemma 3.4. Let $C$ be a nonempty closed convex subset of $H$ and let $f$ be a bifunction of $C \times C$ into $\mathbf{R}$ satisfying (A1)-(A4). Let $\left\{r_{n}\right\}$ be a positive real sequence such that $\lim _{n \rightarrow \infty} r_{n}=r>0$. Then the sequence $\left\{T_{r_{n}}\right\}$ satisfies the $(S U)$ condition.

Proof. (1) Let $\left\{x_{n}\right\}$ be a convergent sequence in C. Let $z_{n}=T_{r_{n}} x_{n}$, for all $n$, then

$$
\begin{gathered}
f\left(z_{n}, y\right)+\frac{1}{r}\left\langle y-z_{n}, z_{n}-x_{n}\right\rangle \geq 0, \quad \forall y \in C, \\
f\left(z_{n+m}, y\right)+\frac{1}{r}\left\langle y-z_{n+m}, z_{n+m}-x_{n+m}\right\rangle \geq 0, \quad \forall y \in C .
\end{gathered}
$$

Putting $y=z_{n+m}$ in (3.6) and $y=z_{n}$ in (3.7), we have

$$
\begin{gathered}
f\left(z_{n}, z_{n+m}\right)+\frac{1}{r}\left\langle z_{n+m}-z_{n}, z_{n}-x_{n}\right\rangle \geq 0, \quad \forall y \in C, \\
f\left(z_{n+m}, z_{n}\right)+\frac{1}{r}\left\langle z_{n}-z_{n+m}, z_{n+m}-x_{n+m}\right\rangle \geq 0, \quad \forall y \in C .
\end{gathered}
$$

So, from (A2) we have

$$
\left\langle z_{n+m}-z_{n}, \frac{z_{n}-x_{n}}{r_{n}}-\frac{z_{n+m}-x_{n+m}}{r_{n+m}}\right\rangle \geq 0,
$$

and hence

$$
\left\langle z_{n+m}-z_{n}, z_{n}-x_{n}-\frac{r_{n}}{r_{n+m}}\left(z_{n+m}-x_{n+m}\right)\right\rangle \geq 0
$$

Thus, we have

$$
\left\langle z_{n+m}-z_{n}, z_{n}-z_{n+m}+z_{n+m}-x_{n}-\frac{r_{n}}{r_{n+m}}\left(z_{n+m}-x_{n+m}\right)\right\rangle \geq 0,
$$

which implies that

$$
\begin{aligned}
\left\|z_{n+m}-z_{n}\right\|^{2} & \leq\left\langle z_{n+m}-z_{n}, z_{n+m}-x_{n}-\frac{r_{n}}{r_{n+m}}\left(z_{n+m}-x_{n+m}\right)\right\rangle \\
& =\left\langle z_{n+m}-z_{n},\left(1-\frac{r_{n}}{r_{n+m}}\right) z_{n+m}+\frac{r_{n}}{r_{n+m}}\left(x_{n+m}-x_{n}\right)\right\rangle .
\end{aligned}
$$


Therefore, we get

$$
\left\|z_{n+m}-z_{n}\right\| \leq\left|1-\frac{r_{n}}{r_{n+m}}\right|\left\|z_{n+m}\right\|+\left\|\frac{r_{n}}{r_{n+m}} x_{n+m}-x_{n}\right\| .
$$

On the other hand, for any $p \in \operatorname{EP}(f)$, from $z_{n}=T_{r_{n}} x_{n}$, we have

$$
\left\|z_{n}-p\right\|=\left\|T_{r_{n}} x_{n}-p\right\| \leq\left\|x_{n}-p\right\|
$$

so that $\left\{z_{n}\right\}$ is bounded. Since $\lim _{n \rightarrow \infty} r_{n}=r>0$, this together with (3.13) implies that the $\left\{z_{n}\right\}$ is a Cauchy sequence. Hence $T_{r_{n}} x_{n}=z_{n}$ is convergent.

(2) By using Lemma 3.2, we know that

$$
\bigcap_{n=0}^{\infty} F\left(T_{r_{n}}\right)=\mathrm{EP}(f) \neq \varnothing
$$

(3) From (1) we know that, $\lim _{n \rightarrow \infty} T_{r_{n}} x$ exists, for all $x \in C$. So, we can define a mapping $T$ from $C$ into itself by

$$
T x=\lim _{n \rightarrow \infty} T_{r_{n}} x, \quad \forall x \in C .
$$

It is obvious that the $T$ is nonexpansive. It is easy to see that

$$
\mathrm{EP}(f)=\bigcap_{n=1}^{\infty} F\left(T_{r_{n}}\right) \subset F(T) .
$$

On the other hand, let $w \in F(T), w_{n}=T_{r_{n}} w$, we have

$$
f\left(w_{n}, y\right)+\frac{1}{r}\left\langle y-w_{n}, w_{n}-w\right\rangle \geq 0, \quad \forall y \in C
$$

By (A2) we know

$$
\frac{1}{r}\left\langle y-w_{n}, w_{n}-w\right\rangle \geq f\left(y, w_{n}\right), \quad \forall y \in C
$$

Since $w_{n} \rightarrow T w=w$ and from (A4), we have $f(y, w) \leq 0$, for all $y \in C$. Then, for $t \in(0,1]$ and $y \in C$,

$$
\begin{aligned}
0 & =f(t y+(1-t) w, t y+(1-t) w) \\
& \leq t f(t y+(1-t) w, y)+(1-t) f(t y+(1-t) w, w) \\
& \leq t f(t y+(1-t) w, y) .
\end{aligned}
$$


Therefore, we have

$$
f(t y+(1-t) w, y) \geq 0
$$

Letting $t \downarrow 0$ and using (A3), we get

$$
f(w, y) \geq 0, \quad \forall y \in C
$$

and hence $w \in \operatorname{EP}(f)$. From the aforementioned two respects, we know that $F(T)=$ $\bigcap_{n=0}^{\infty} F\left(T_{r_{n}}\right)$. This completes the proof.

Theorem 3.5. Let $C$ be a nonempty closed convex subset of a real Hilbert space $H$. Let $f$ be a bifunction from $C \times C$ into $R=(-\infty,+\infty)$ satisfying $(A 1)-(A 4)$ and $E P(f) \neq \varnothing$. Let $\left\{x_{n}\right\}$ and $\left\{u_{n}\right\}$ be sequences generated by $x_{0} \in C$ and

$$
\begin{gathered}
x_{0} \in C \text { chosen arbitrarily, } \\
f\left(u_{n}, x_{n}\right)+\frac{1}{r_{n}}\left\langle y-u_{n}, u_{n}-x_{n}\right\rangle \leq 0, \quad \forall y \in C, \\
y_{n}=\alpha_{n} x_{n}+\left(1-\alpha_{n}\right) u_{n}, \\
C_{n}=\left\{z \in C_{n-1} \bigcap Q_{n-1}:\left\|y_{n}-z\right\| \leq\left\|x_{n}-z\right\|\right\}, \quad n \geq 1, \\
C_{0}=\left\{z \in C:\left\|y_{0}-z\right\| \leq\left\|x_{0}-z\right\|\right\}, \\
Q_{n}=\left\{z \in C_{n-1} \bigcap Q_{n-1}:\left\langle x_{n}-z, x_{0}-x_{n}\right\rangle \geq 0\right\}, \quad n \geq 1, \\
Q_{0}=C, \\
x_{n+1}=P_{C_{n} \cap Q_{n}}\left(x_{0}\right) .
\end{gathered}
$$

Assume $\lim _{n \rightarrow \infty} r_{n}=r>0$ and $\limsup _{n \rightarrow \infty} \alpha_{n}<1$. Then $\left\{x_{n}\right\}$ converges strongly to $P_{E P(f)} x_{0}$, where $P_{E P(f)}$ is the metric projection from $\mathrm{H}$ onto $\operatorname{EP}(f)$.

Proof. By using Lemma 3.4, we know that $\left\{T_{r_{n}}\right\}$ satisfies the (SU) condition. Then $F(T)=$ $\mathrm{EP}(f)$. By using Theorem 2.2, we obtain the result of Theorem 3.5

Now, we study a kind of optimization problem by using the aforementioned results of this paper. That is, we will give an iterative algorithm of solution for the following optimization problem with nonempty set of solutions:

$$
\begin{gathered}
\min h(x), \\
x \in C,
\end{gathered}
$$

where $h(x)$ is a convex and lower semicontinuous functional defined on a closed convex subset $C$ of a Hilbert space $H$. We denoted by $A$ the set of solutions of (3.24). Let $f$ be a 
bifunction from $C \times C$ to $R$ defined by $f(x, y)=h(y)-h(x)$. We consider the following equilibrium problem, that is to find $x \in C$ such that

$$
f(x, y) \geq 0, \quad \forall y \in C
$$

It is obvious that $\mathrm{EP}(F)=A$, where $\mathrm{EP}(F)$ denotes the set of solutions of equilibrium Problem (3.25). In addition, it is easy to see that $f(x, y)$ satisfies the conditions (A1)-(A4) in Section 2. Therefore, from Theorem 3.5, we can obtain the following theorem.

Theorem 3.6. Let $C$ be a nonempty closed convex subset of a real Hilbert space $H$. Let $h(x)$ be a convex and lower semicontinuous functional defined on $C$. Let $\left\{x_{n}\right\}$ and $\left\{u_{n}\right\}$ be sequences generated by $x_{0} \in C$ and

$$
\begin{gathered}
x_{0} \in C \text { chosen arbitrarily, } \\
h\left(x_{n}\right)-h\left(u_{n}\right)+\frac{1}{r_{n}}\left\langle y-u_{n}, u_{n}-x_{n}\right\rangle \leq 0, \quad \forall y \in C, \\
y_{n}=\alpha_{n} x_{n}+\left(1-\alpha_{n}\right) u_{n}, \\
C_{n}=\left\{z \in C_{n-1} \bigcap Q_{n-1}:\left\|y_{n}-z\right\| \leq\left\|x_{n}-z\right\|\right\}, \quad n \geq 1, \\
C_{0}=\left\{z \in C:\left\|y_{0}-z\right\| \leq\left\|x_{0}-z\right\|\right\}, \\
Q_{n}=\left\{z \in C_{n-1} \bigcap Q_{n-1}:\left\langle x_{n}-z, x_{0}-x_{n}\right\rangle \geq 0\right\}, \quad n \geq 1, \\
Q_{0}=C, \\
x_{n+1}=P_{C_{n} \cap Q_{n}}\left(x_{0}\right) .
\end{gathered}
$$

Assume $\lim _{n \rightarrow \infty} r_{n}=r>0$ and $\lim \sup _{n \rightarrow \infty} \alpha_{n}<1$. Then $\left\{x_{n}\right\}$ converges strongly to $P_{A} x_{0}$.

Remark 3.7. It is easy to see that this paper has some new methods and results than the results of Nilsrakoo and Saejung [11]:

(1) proposed a modified hybrid iterative scheme, so that the new simple method of proof has been used;

(2) removed the bounded restriction for closed convex set $C$;

(3) relax the conditions of sequence $\left\{T_{n}\right\}$;

(4) give an application for optimization problem;

(5) the sequence of sets $C_{n}, Q_{n}$ satisfy the following relation:

$$
F(T) \subset \cdots C_{n+1} \cap Q_{n+1} \subset C_{n} \cap Q_{n}, \quad n \geq 0,
$$

so that to raise the convergence rate of $\left\{x_{n}\right\}$ is possible. 


\section{Acknowledgment}

This project is supported by the National Natural Science Foundation of China under $\operatorname{grant}(10771050)$.

\section{References}

[1] W. R. Mann, "Mean value methods in iteration," Proceedings of the American Mathematical Society, vol. 4, no. 3, pp. 506-510, 1953.

[2] X. Qin and Y. Su, "Strong convergence theorems for relatively nonexpansive mappings in a Banach space," Nonlinear Analysis: Theory, Methods E Applications, vol. 67, no. 6, pp. 1958-1965, 2007.

[3] A. Genel and J. Lindenstrauss, "An example concerning fixed points," Israel Journal of Mathematics, vol. 22, no. 1, pp. 81-86, 1975.

[4] K. Nakajo and W. Takahashi, "Strong convergence theorems for nonexpansive mappings and nonexpansive semigroups," Journal of Mathematical Analysis and Applications, vol. 279, no. 2, pp. 372$379,2003$.

[5] T.-H. Kim and H.-K. Xu, "Strong convergence of modified Mann iterations for asymptotically nonexpansive mappings and semigroups," Nonlinear Analysis: Theory, Methods \& Applications, vol. 64, no. 5, pp. 1140-1152, 2006.

[6] K. Nakajo, K. Shimoji, and W. Takahashi, "Strong convergence theorems by the hybrid method for families of nonexpansive mappings in Hilbert spaces," Taiwanese Journal of Mathematics, vol. 10, no. 2, pp. 339-360, 2006.

[7] Y. Su and X. Qin, "Monotone CQ iteration processes for nonexpansive semigroups and maximal monotone operators," Nonlinear Analysis: Theory, Methods E Applications, vol. 68, no. 12, pp. 36573664, 2008.

[8] Y. Su, D. Wang, and M. Shang, "Strong convergence of monotone hybrid algorithm for hemi-relatively nonexpansive mappings," Fixed Point Theory and Applications, vol. 2008, Article ID 284613, 8 pages, 2008.

[9] W. Takahashi and K. Zembayashi, "Strong convergence theorem by a new hybrid method for equilibrium problems and relatively nonexpansive mappings," Fixed Point Theory and Applications, vol. 2008, Article ID 528476, 11 pages, 2008.

[10] Y. Cheng and M. Tian, "Strong convergence theorem by monotone hybrid algorithm for equilibrium problems, hemirelatively nonexpansive mappings, and maximal monotone operators," Fixed Point Theory and Applications, vol. 2008, Article ID 617248, 12 pages, 2008.

[11] W. Nilsrakoo and S. Saejung, "Weak and strong convergence theorems for countable Lipschitzian mappings and its applications," Nonlinear Analysis: Theory, Methods E Applications, vol. 69, no. 8, pp. 2695-2708, 2008.

[12] E. Blum and W. Oettli, "From optimization and variational inequalities to equilibrium problems," The Mathematics Student, vol. 63, no. 1-4, pp. 123-145, 1994.

[13] P. L. Combettes and S. A. Hirstoaga, "Equilibrium programming in Hilbert spaces," Journal of Nonlinear and Convex Analysis, vol. 6, no. 1, pp. 117-136, 2005.

[14] S. D. Flåm and A. S. Antipin, "Equilibrium programming using proximal-like algorithms," Mathematical Programming, vol. 78, no. 1, pp. 29-41, 1997.

[15] A. Moudafi and M. Théra, "Proximal and dynamical approaches to equilibrium problems," in Ill-Posed Variational Problems and Regularization Techniques (Trier, 1998), vol. 477 of Lecture Notes in Economics and Mathematical Systems, pp. 187-201, Springer, Berlin, Germany, 1999.

[16] S. Takahashi and W. Takahashi, "Viscosity approximation methods for equilibrium problems and fixed point problems in Hilbert spaces," Journal of Mathematical Analysis and Applications, vol. 331, no. 1 , pp. 506-515, 2007. 